\title{
The Nipple is Just Another Margin
}

\author{
Suzanne B. Coopey, MD and Barbara L. Smith, MD, PhD \\ Division of Surgical Oncology, Massachusetts General Hospital, Harvard Medical School, Boston
}

The indications for nipple-sparing mastectomy (NSM), also termed "total skin-sparing mastectomy," have evolved to include the majority of patients undergoing mastectomy. Few absolute contraindications to nipple sparing remain. These have included direct involvement of the nippleareola complex (NAC), observed on preoperative clinical exam or imaging, or involvement of the nipple margin tissue seen on pathology.

At some centers, patients with locally advanced breast cancer are considered eligible for nipple sparing after neoadjuvant systemic therapy and with postmastectomy radiation. Cosmetic contraindications to nipple sparing include factors that would result in an unacceptable position of the retained nipple on the reconstructed breast, such as marked breast ptosis or very large breast size.

As use of NSM increases, algorithms for management of close or positive nipple margins are needed. Historical rates of positive nipple margins in nipple-sacrificing mastectomies are as high as $50 \%$, with a $21 \%$ rate of tumor involvement of nipples in mastectomies performed at our institution in 2006. ${ }^{1}$ Despite these high rates of nipple involvement in standard mastectomy series, rates of positive nipple margins in modern NSM series are low, likely due to appropriate patient selection.

In this issue of Annals of Surgical Oncology, Amara et al. $^{2}$ report their experience managing positive nipple margins in their series of 1173 NSMs from 2005 to 2013. They report a positive nipple margin rate of $2.7 \%$ among all NSM. When the 440 prophylactic NSMs are excluded, their positive nipple margin rate for therapeutic mastectomies is $4.4 \%$. In other series, rates of positive nipple margins in therapeutic NSM range from 2.5 to $10 \% .^{3-8}$ To

(C) Society of Surgical Oncology 2015

First Received: 7 April 2015;

Published Online: 3 June 2015

S. B. Coopey, MD

e-mail: scoopey@partners.org date, little has been written about the management of positive nipple margins in NSM patients, and Amara et al. add their large patient cohort to this field. ${ }^{7,8}$

In considering management options for positive nipple margins, it is important to define "nipple margin" and the appropriate technique for obtaining an adequate sample. Many techniques have been described. Amara et al. ${ }^{2}$ perform inversion of the nipple and excision of the nipple tissue at the dermal junction, then core out the inside of the nipple as a separate specimen. They then mark the subareolar margin, deep only to the NAC on the mastectomy specimen, for pathologic examination. They consider any tumor involvement of either the marked subareolar tissue or the nipple core as a positive nipple margin. It is unclear how much subareolar tissue actually undergoes pathologic evaluation with this approach.

Our group previously described ex vivo analysis of nipple margin assessment techniques in nipple-sacrificing mastectomy specimens. ${ }^{9}$ When a technique similar to that of Amara et al. ${ }^{2}$ was used, sharply dividing breast tissue from under NAC skin and marking the subareolar tissue for analysis, the subareolar tissue specimen did not reliably contain ductal tissue. Rusby et al. ${ }^{9}$ hypothesized that the divided duct bundle retracted into the mastectomy specimen and therefore was not present in the sampled tissue from the surface of the mastectomy specimen.

A revised technique adopted by our group involves raising areola skin flaps, leaving the nipple duct bundle intact, grasping the duct bundle with a curved clamp immediately below the NAC dermis, sharply dividing the tissue along both sides of the clamp, and sending the contents of this clamp as the nipple margin specimen. This technique yields many more ducts in a small specimen that is completely submitted for pathologic analysis. This approach also completely clears the nipple-areola skin of fat, breast parenchyma, and ductal tissue, eliminating the need for additional sharp dissection within the nipple papilla and resulting in very low rates of nipple necrosis. ${ }^{10}$ 
Histologic assessment of the nipple margin specimen may be performed on frozen section, which allows for immediate management of the positive margin, or on permanent section. When permanent section is used, the management of the positive nipple margin can be determined in the context of complete pathologic staging and with knowledge of the full treatment plan. Accurate nipple margin assessment on frozen section is difficult, and distinguishing benign atypia from intraductal carcinoma can be challenging, potentially resulting in unnecessary nipple excision. As Amara et al. ${ }^{2}$ and Rusby et al. ${ }^{9}$ suggest, permanent section analysis of nipple margins appears to be the best strategy for maximizing nipple preservation.

When a nipple margin specimen from an NSM is found to contain tumor, management options include complete excision of the NAC, full-thickness excision of the nipple with preservation of the areola (not described in the article of Amara et al. $^{2}$ ), radiation of the retained nipple, or observation alone, with or without systemic therapy. Amara et al. $^{2}$ add the option of re-excising the nipple margin with preservation of nipple skin. However, if the nipple margin is obtained in the manner described by Amara et al. ${ }^{2}$ or Rusby et al. ${ }^{9}$ there should be no residual nipple tissue or subareolar breast tissue to re-excise, and it seems possible that re-coring the nipple could compromise nipple viability, making this option less attractive.

The majority of excised NAC, nipples, and nipple core re-excisions show no residual tumor, prompting exploration of alternatives to nipple/NAC excision when smallvolume disease is seen in the nipple margin specimen. ${ }^{2,7,8}$ Postmastectomy radiation is a reasonable option for patients requiring radiation for other indications (i.e., tumor $>5 \mathrm{~cm}$, positive lymph nodes, or positive mastectomy specimen margins). Use of a single dose of intraoperative radiation (ELIOT) to the NAC for NSM patients has been described by Petit et al. ${ }^{6}$ Among 1001 patients, these authors observed no NAC recurrences during a median follow-up period of 20 months, even in the 160 patients with close or positive nipple margins. However, by a follow-up visit at a median of 50 months, 861 patients who underwent NSM with ELIOT had experienced 11 NAC recurrences $(1 \%) .{ }^{11}$ Seven of these NAC recurrences were Paget's disease, and all of the Paget's recurrences were experienced by patients with negative nipple margins on the final pathology. These results suggest that radiating the nipple is safe, at least with short-term follow-up evaluation. It is important to note that the surgical technique of Petit et al. ${ }^{6}$ differs from that of Amara et al. ${ }^{2}$ in that a thin layer of glandular tissue $5 \mathrm{~mm}$ thick beneath the areola is routinely left to prevent nipple necrosis, which could explain their higher NAC recurrence rate.

Is it safe to omit further treatment for patients with a positive nipple margin? In certain situations when the nipple margin contains minimal disease, as in patients with "single-focus" or "scant" ductal carcinoma in situ (DCIS), this may seem reasonable. However, when Camp et al. ${ }^{8}$ examined NSMs with positive nipple margins (with more than half having minimal disease in the margin specimen), $24 \%$ contained residual malignancy in the excised nipple or NAC specimen. Although it is encouraging that Amara et al. $^{2}$ report no nipple recurrences after a mean follow-up period of 31.3 months, the observation of increasing recurrences over time by Petit et al. ${ }^{6}$ suggests that additional follow-up time is needed before positive margins are routinely left untreated.

We previously reported on the management of 22 positive nipple margins in the first 438 NSMs at our institution and recently updated our experience managing 46 positive margins in 1326 NSMs. ${ }^{7,8}$ Like Amara et al. ${ }^{2}$ we have moved away from routine excision of the entire NAC. We most frequently perform full-thickness excision of the nipple with retention of the areola, but have occasionally used radiation or observation only in selected cases. Like Amara et al. ${ }^{2}$ we have had no recurrences in the nipple, the areola, or the incision used for excision of a nipple or NAC.

As NSM gains popularity, the dilemma of what to do with the positive nipple margin is an important one. As techniques evolve that allow for staining and/or orienting of the nipple margin, if "no ink on tumor" is obtained, this likely would be sufficient in keeping with recent margin guidelines. Although we recognize that the margin consensus guidelines were designed for patients undergoing breast conservation, with modern systemic therapy and increased use of postmastectomy radiation therapy, it is reasonable to consider the nipple margin of an NSM as just another margin. For now, it is ideal to obtain negative nipple margins until long-term follow-up evaluation of large series such as that of Amara et al. ${ }^{2}$ are available. As the data mature, we may find that alternatives to nipple excision, such as radiation or observation, are safe options as well.

DISCLOSURE None.

\section{REFERENCES}

1. Brachtel EF, Rusby JE, Michaelson JS, et al. Occult nipple involvement in breast cancer: clinicopathologic findings in 316 consecutive mastectomy specimens. J Clin Oncol. 2009;27:4948-54.

2. Amara D, Peled AW, Wang F, et al. Tumor involvement of the nipple in total skin-sparing mastectomy: strategies for management. Ann Surg Oncol. 2015. doi:10.1245/s10434-015-4646-5.

3. Crowe JP, Patrick RJ, Yetman RJ, Djohan R. Nipple-sparing mastectomy update: one hundred forty-nine procedures and clinical outcomes. Arch Surg. 2008;14311:1106-10.

4. Boneti C, Yuen J, Santiago C, et al. Oncologic safety of nipple skin-sparing or total skin-sparing mastectomies with immediate reconstruction. J Am Coll Surg. 2011;212:686-95. 
5. Filho PA, Capko D, Barry JM, et al. Nipple-sparing mastectomy for breast cancer and risk-reducing surgery: the Memorial SloanKettering Cancer Center experience. Ann Surg Oncol. 2011;18:3117-22.

6. Petit JY, Veronisi U, Orecchia R, et al. Nipple-sparing mastectomy with nipple-areola intraoperative radiotherapy: one thousand and one cases of a five years experience at the European institute of oncology of Milan (EIO). Breast Cancer Res Treat. 2009;117:333-8.

7. Tang R, Coopey SB, Merrill, et al. Positive subareolar/nipple margins in nipple-sparing mastectomies: rates, management, and oncologist safety (abstract). ASBD 2015.

8. Camp MS, Coopey SB, Tang R, et al. Management of positive subareolar/nipple margins in nipple-sparing mastectomies. Breast J. 2014;20:402-7.
9. Rusby JE, Kirstein LJ, Brachtel EF, et al. Nipple-sparing mastectomy: lessons from ex vivo procedures. Breast $J$. 2008; 14:464-70.

10. Coopey SB, Tang R, Lei L, et al. Increasing eligibility for nipplesparing mastectomy. Ann Surg Oncol. 2013;20:3218-22.

11. Lohsiriwat V, Martella S, Rietjens M, et al. Paget's disease as a local recurrence after nipple-sparing mastectomy: clinical presentation, treatment, outcome, and risk factor analysis. Ann Surg Oncol. 2012;19:1850-5. 